
internationales

vol. 34 - $n^{\circ} 1 \mid 2018$

Mouvements migratoires d'hier et d'aujourd'hui en Italie

\title{
Les migrations transocéaniques italiennes au début du XXe siècle : un autre regard
}

Italian Transoceanic Migration at the Beginning of the 20th Century: A Different Look

La migración transoceánica italiana a principios del siglo XX: una otra mirada

\section{Augusta Molinari}

\section{OpenEdition}

Journals

Édition électronique

URL : https://journals.openedition.org/remi/10069

DOI : 10.4000/remi. 10069

ISSN : $1777-5418$

Éditeur

Université de Poitiers

Édition imprimée

Date de publication : 1 avril 2018

Pagination : 79-101

ISBN : 979-10-90426-61-0

ISSN : 0765-0752

Référence électronique

Augusta Molinari, «Les migrations transocéaniques italiennes au début du XXe siècle : un autre regard », Revue européenne des migrations internationales [En ligne], vol. $34-n^{\circ} 1$ | 2018, mis en ligne le 28 décembre 2019, consulté le 19 avril 2022. URL : http://journals.openedition.org/remi/10069 ; DOI https://doi.org/10.4000/remi.10069 


\section{Les migrations transocéaniques italiennes au début du XXe siècle: un autre regard}

\section{Augusta Molinari ${ }^{1}$}

\section{Les frontières de la mer}

Les morts qui se succèdent depuis vingt ans dans le canal de Sicile sont le résultat le plus dramatique d'une législation européenne et nationale qui a fait du franchissement des frontières en mer un acte illégal (Sossi, 2005; Cuttitta, 2012; Leogrande, 2015). La traversée de la Méditerranée est de plus en plus mise en scène ; elle est presque devenue une forme de survie, résultat d'actions de violence et de cruauté qui ont déterminé la redéfinition d'une nouvelle géopolitique au niveau mondial (Dal Lago, 2003) qui s'est mise en place entre le XXe et le XXle siècle. Le voyage en mer constitue désormais une violation de frontières que la mondialisation a créé et qui est devenu toujours plus omniprésent (Schmoll et al., 2015). Ces passages tragiques nous invitent à porter un regard différent sur les migrations transocéaniques italiennes du début du XXe siècle : le fait de franchir une frontière au moment du débarquement a été peu étudié (Neuman, 1996; Tichenor, 2002; Kanstroom, 2007). À leur arrivée, des migrants italiens pouvaient être refoulés ou subir un rapatriement forcé des États-Unis vers I'Italie, comme le montrent les rares données du Commissariato generale dell'emigrazione $^{2}$ (1926) l'organe qui a traité en Italie la gestion des flux migratoires. Cette "comptabilité " du désespoir, de la misère, de la défaite relative aux voyages de retour des émigrants n'a donc laissé que peu de traces dans les statistiques.

\section{Les bateaux-lazarets}

Le départ de navires chargés de migrants se dirigeant vers les Amériques a constitué entre 1850 et 1920 le symbole des migrations transocéaniques italiennes. L'iconographie, les journaux, les chants populaires parlent des "navires qui partent" et évoquent la complexité et la variété des expériences migratoires. La clameur qui accompagne le départ des émigrants pour les

1 Professeure d'histoire contemporaine, Université de Gênes, Département des sciences de I'éducation, Corso Andrea Podestà 18100 Gênes, Italie ; augusta.molinari@unige.it

2 Commissariat général de l'émigration. 
Amériques est motivée par le caractère de la séparation définitive du pays d'origine attribuée par la classe politique de l'époque aux migrations transocéaniques. Les flux migratoires vers les Amériques sont présentés comme des migrations sans retour qui "libèrent" la société italienne du poids de sujets économiquement inutiles et socialement dangereux (Sori, 1979; Arru et Ramella, 2003; Orazi, 2015). D'ailleurs, une fois à bord des navires, les migrants n'avaient plus aucune forme de protection de la part de l'État italien. C'est seulement à partir de $1901^{3}$ avec la première loi sur l'émigration que le gouvernement italien prévoit la présence d'un représentant de l'État, un médecin officier de la Marine (le Commissaire de bord), sur les navires qui transportaient les migrants. Les Commissaires étaient chargés de vérifier les conditions sanitaires et d'hygiène de vie des migrants sur les navires pendant la traversée (Molinari, 2014a). Mais, à cause des multiples tâches qui leur étaient attribuées et de leur nombre insuffisant pour répondre aux exigences du transport des migrants, la présence à bord de ces officiers n'a pas produit une amélioration des conditions de voyage des migrants, tout du moins jusqu'aux années 1920.

Pour les migrants italiens, traverser l'océan était un passage normal et habituel, bien qu'à cette époque les traversées n'étaient pas dénuées de risques. Durant le voyage, les migrants pouvaient décéder, tomber gravement malades, être victimes de naufrage ou bien ils pouvaient être débarqués dans des lieux différents que ceux prévus, être rejetés au moment du débarquement dans les ports d'arrivée, être rapatriés parce qu'ils étaient malades ou en raison de leur pauvreté.

Le voyage d'émigration représente un des rares éléments d'observation permettant de recueillir les données qualitatives et quantitatives sur les conditions de santé des migrations historiques italiennes transocéaniques. Les bateaux, en effet, apparaissent en soi comme un lieu pathogène entre le XIXe et le XXe siècle (Molinari, 2005).

L'institution en 1901 du Commissariat général de l'émigration (Ostuni, 2001) créé une structure politique administrative dont dépend une série de services destinés au contrôle sanitaire des migrants avant l'embarquement et au cours du voyage. Les informations enregistrées nous permettent d'avoir une ample documentation sur les pathologies les plus fréquentes rencontrées à bord des navires, le type d'assistance sanitaire apportée et l'hygiène à bord des bateaux à vapeur. II s'agit d'un corpus hétérogène (rapports de santé préparés par le Commissariat général, dossiers sur la santé rédigés par le médecin du gouvernement embarqué sur les bateaux, rapports des médecins à bord, statistiques sanitaires préparées par le Commissariat général de l'émigration, enquêtes et publications de médecins et d'hygiénistes sur les conditions sanitaires des voyages transocéaniques) d'un grand intérêt pour documenter les conditions hygiéniques et sanitaires des voyages au début du XXe siècle (Molinari, 1990, 1993 et 2004). La majorité de cette documentation est conservée par les archives

\footnotetext{
3 La loi de 1901, avec l'instauration du Commissariat général de l'émigration, prévoyait une série de dispositifs de contrôle et de protection des migrations transocéaniques (inspection de l'émigration dans les ports d'embarquement, hospitalisation des migrants en attente $d^{\prime}$ embarquement, conventions avec les auberges où logeaient les migrants en attente du départ) qui ne furent appliqués que partiellement après 1910.
} 
centrales de l'État et regroupée avec le fonds du ministère de l'Intérieur ${ }^{4}$. L'ensemble de ces documents couvre une période qui va de la dernière décennie du XIXe siècle à la suppression, en 1927, du Commissariat général de l'émigration.

Le transport en masse d'émigrants par la mer sur les voies transocéaniques représente, en effet, une situation à haut risque pour la santé, non seulement en raison des mauvaises conditions d'hygiène régnant à bord et de l'inadaptation des structures et du personnel sanitaire pour répondre aux multiples besoins d'assistance, mais aussi en raison des urgences fréquentes dues à l'apparition d'épidémies au cours du voyage. Le manque de structures sanitaires sur les bateaux réservés à l'émigration, le surpeuplement et le manque d'hygiène avec lesquels les émigrants étaient contraints de voyager, font partie des éléments récurrents des rapports sanitaires établis par les médecins les plus scrupuleux. La situation sanitaire à bord des bateaux à vapeur, déjà difficile à gérer dans un contexte normal, devenait insoutenable quand, ce qui arrivait assez fréquemment, se manifestaient des épidémies de maladies infectieuses.

Le médecin du gouvernement du bateau Città di Torino, présente une situation sanitaire à haut risque au cours d'une traversée à destination de New York en décembre 1906 :

"Les conditions sanitaires du voyage à l'aller ont été peu satisfaisantes : sur

1401 émigrants, quarante-huit ont été soignés à l'infirmerie de bord, dont trois seulement ont guéri durant la traversée, quarante-quatre ont débarqué malades et un a trouvé la mort. Les causes d'un taux aussi élevé de morbidité doivent être attribuées à trois maladies infectieuses qui se sont développées simultanément à bord: la variole, la varicelle et la rougeole. $n^{5}$

Dans la description des mesures sanitaires adoptées pour contenir les épisodes épidémiques, le médecin souligne les difficultés rencontrées afin d'isoler les malades contagieux en raison du manque de locaux adaptés.

Si la succession d'urgences sanitaires importantes au fil des traversées, favorisées par les conditions de surpeuplement, de saleté et de violence que subissaient les émigrants, mettait à dure épreuve un service sanitaire déjà très peu efficace, ces situations difficilement soutenables conduisaient les médecins à prendre des positions de dénonciations explicites du danger que constituait un voyage transocéanique pour la santé des émigrants. Comme nous pouvons le lire dans la conclusion du rapport, concis, mais efficace, à propos du voyage de retour des États-Unis à bord du bateau Calabria au mois de février 1903 :

"Il y avait des tuberculeux, des malades atteints du paludisme et de rhumatismes chroniques. Durant le voyage de retour, le bateau à vapeur ressemblait à une grande infirmerie. $1^{6}$

\footnotetext{
4 ACS (Archivio Centrale dello Stato), MI (Ministero degli Interni) DGSP (Direzione Generale di Sanità Pubblica) (1882-1927) Relazioni sanitarie, bb. 12-198.

5 ACS, MI, DGSP (1882-1915) Giornali sanitari. Giornale sanitario del piroscafo Città di Torino, b. 168.

6 ACS, MI, DGSP (1900-1914) Giornali sanitari. Giornale sanitario del piroscafo Calabria, b. 8.
} 
Tableau 1 : Cas de maladies infectieuses et d'aliénations mentales déclarés durant les voyages transocéaniques en effectifs (1903-1925)

\begin{tabular}{|c|c|c|c|c|}
\hline & $\begin{array}{c}\text { Italie - Amérique } \\
\text { du Nord }\end{array}$ & $\begin{array}{l}\text { Amérique du } \\
\text { Nord - Italie }\end{array}$ & $\begin{array}{c}\text { Italie - Amérique } \\
\text { du Sud }\end{array}$ & $\begin{array}{l}\text { Amérique du } \\
\text { Sud - Italie }\end{array}$ \\
\hline Ankylostome & 0 & 11 & 0 & 152 \\
\hline Paludisme & 162 & 57 & 133 & 46 \\
\hline Rougeole & 1695 & 880 & 2559 & 1310 \\
\hline Pneumonie & 403 & 119 & 349 & 89 \\
\hline Syphilis & 33 & 232 & 106 & 223 \\
\hline Trachome & 167 & 677 & 415 & 2623 \\
\hline $\begin{array}{l}\text { Tuberculose } \\
\text { pulmonaire }\end{array}$ & 143 & 7745 & 213 & 1929 \\
\hline Gale & 395 & 344 & 508 & 275 \\
\hline Variole & 24 & 13 & 50 & 44 \\
\hline Scarlatine & 52 & 37 & 49 & 70 \\
\hline $\begin{array}{l}\text { Variole } \\
\text { mineure }\end{array}$ & 122 & 26 & 75 & 29 \\
\hline Typhoïde & 217 & 93 & 159 & 88 \\
\hline Varicelle & 278 & 479 & 327 & 159 \\
\hline $\begin{array}{l}\text { Aliénation } \\
\text { mentale }\end{array}$ & 92 & 2237 & 150 & 748 \\
\hline
\end{tabular}

Source : Commissariato generale dell'emigrazione (1926 : 1613-1628).

La prédominance des malades atteints de tuberculose pulmonaire parmi les patients soignés dans les hôpitaux de bord constitue une donnée récurrente aussi bien dans les rapports sanitaires examinés (voir Tableau 1) qui font référence à des voyages réalisés dans la première décennie du $\mathrm{XXe}$ siècle, que dans les journaux sanitaires consultés sur une période allant de 1925 à 1928. Le pourcentage des émigrants refoulés dans les ports de débarquement, puis rapatriés pour tuberculose pulmonaire n'est pas indiqué dans les statistiques sanitaires du Commissariat général de l'émigration qui se limitent à relever le nombre total des immigrants refoulés et des rapatriés inscrits comme indigents, mais ce chiffre ressort de l'analyse des documents sanitaires.

Pour les médecins, la présence de nombreux tuberculeux sur les bateaux au retour, surtout, des États-Unis, était considérée comme faisant partie de la routine (voir Tableau 2):

"Enfin, d'autres passagers, bien qu'ils n'aient pas été hospitalisés, ont été quand même correctement soignés en ambulatoire et ont eu droit à une nourriture spéciale, sachant que de nombreux rapatriés d'Amérique du Nord retournent auprès de leur famille atteints de lésions chroniques, notamment à l'appareil respiratoire. $n^{7}$

Lorsque le médecin du bateau Republic dresse son rapport sur le nombre de malades durant le retour de New York à Naples en février 1906, ce dernier ne réussit pas à taire son jugement sévère à propos des conditions d'exploitation auxquelles étaient soumis les émigrants italiens aux États-Unis :

7 ACS, MI, DGSP (1882-1915) Giornali sanitari. Giornale sanitario Duca degli Abruzzi, b. 45. 
"Remarquable, comme toujours, le pourcentage de malades chroniques et d'invalides que l'Amérique nous renvoie, sans même avoir auparavant donné un minimum de compensation pécuniaire à ceux qui sont dans cet état. $1^{8}$

Tableau 2 : Morbidité et mortalité dues à la tuberculose durant les traversées transocéaniques entre l'Italie et l'Amérique du Nord (1903-1906)

\begin{tabular}{lcccc}
\hline & $\begin{array}{c}\text { Voyageurs } \\
(\mathrm{n})\end{array}$ & $\begin{array}{c}\text { Incidence } \\
(\mathrm{n})\end{array}$ & $\begin{array}{c}\text { Incidence } \\
(\%)\end{array}$ & $\begin{array}{c}\text { Décès } \\
(\mathrm{n})\end{array}$ \\
\hline $\begin{array}{l}\text { Italie - Amérique } \\
\text { du Nord }\end{array}$ & & & & \\
1903 & 177049 & 2 & 0 & 0 \\
1904 & 132534 & 0 & 0 & 0 \\
1905 & 222679 & 18 & 0,08 & 0 \\
1906 & 278316 & 8 & 0,03 & 0 \\
\hline Amérique du & & & & \\
Nord - Italie & & & & \\
1903 & 61203 & 129 & 2,10 & 9 \\
1904 & 108026 & 278 & 2,57 & 9 \\
1905 & 65145 & 369 & 5,66 & 7 \\
1906 & 81412 & 457 & 5,61 & 16 \\
\hline
\end{tabular}

Sources : Padovani (1909 : 21); ACS, MI, DGSP (1900-1915) Giornali sanitari.

Parallèlement au pourcentage élevé de tuberculeux, les bateaux à vapeur qui provenaient des États-Unis transportaient également de nombreux émigrants présentant des formes d'aliénation mentale. Ce type de pathologie était considéré déjà au début $d u X X e$ siècle par le règlement américain comme une cause de rejet et de déportation (Molinari, 2010 et 2014b; Campesi, 2013).

Si les données fournies par les statistiques du Commissariat sur les maladies des émigrants durant les voyages de retour de l'Amérique du Nord, bien que sous-estimant l'ampleur des pathologies les plus diffuses, permettent de saisir les pathologies, il n'est pas possible d'utiliser le même procédé pour les rapatriés d'Amérique du Sud. En effet, les données sanitaires relatives aux voyages allerretour vers l'Amérique du Sud laissent apparaître une forte prédominance du trachome durant les voyages de retour, mais aussi, dans une moindre mesure, la présence de la tuberculose et du paludisme (voir Tableau 3).

Il faut souligner qu'au début du XXe siècle, le trachome était une maladie très fréquente dans certaines régions d'Italie (Pouilles, Sicile, Sardaigne) comme nous le constatons au regard du nombre élevé de personnes atteintes de trachome refoulées lors de l'embarquement pour les États-Unis dans les ports italiens (Rosati, 1909). La présence élevée de personnes atteintes de trachome parmi les Italiens immigrés en Amérique du Sud est donc le résultat de plusieurs facteurs relatifs aussi bien aux conditions d'insertion dans le marché du travail du pays d'immigration, qu'aux modalités de recrutement et de transport des émigrants. 
Tableau 3 : Incidence des maladies lors des traversées transocéaniques entre l'Italie et l'Amérique du Sud (nombre de nouveaux cas pour mille voyageurs, 1903-1925)

\begin{tabular}{|c|c|c|c|c|c|}
\hline & Paludisme & Rougeole & $\begin{array}{l}\text { Maladies bronco- } \\
\text { pulmonaires }\end{array}$ & Trachome & Tuberculose \\
\hline 1903 & 0,66 & 2,35 & 0,39 & 0 & 1,51 \\
\hline 1904 & 0,88 & 0,26 & 0,42 & 0 & 1,78 \\
\hline 1905 & 1,08 & 2,02 & 0,46 & 0 & 1,80 \\
\hline 1906 & 0,77 & 2,21 & 0,87 & 0 & 1,65 \\
\hline 1907 & 1,23 & 2,23 & 0,62 & 0 & 1,74 \\
\hline 1908 & 1,10 & 1,62 & 0,20 & 4,29 & 2,50 \\
\hline 1909 & 0,64 & 1,69 & 0,10 & 0,29 & 2,11 \\
\hline 1910 & 1,49 & 1,19 & 0,06 & 11,31 & 2,66 \\
\hline 1911 & 0,30 & 1,78 & 0,08 & 7,26 & 2,79 \\
\hline 1912 & 0,35 & 1,51 & 0,24 & 4,19 & 1,90 \\
\hline 1913 & 0,57 & 2,91 & 0,09 & 6,71 & 2,55 \\
\hline 1914 & 0,22 & 1,02 & 0,03 & 5,67 & 2,17 \\
\hline 1915 & 0,10 & 2,40 & 0 & 2,15 & 1,47 \\
\hline 1916 & 0,29 & 1,44 & 0 & 2,80 & 3,13 \\
\hline 1917 & 0 & 2,63 & 0 & 2,67 & 3,82 \\
\hline 1918 & 0 & 37,97 & 0 & 7,12 & 1,19 \\
\hline 1919 & 5,22 & 3,01 & 0 & 1,96 & 1,82 \\
\hline 1920 & 1,47 & 3,94 & 0 & 0,05 & 1,38 \\
\hline 1921 & 1,07 & 1,42 & 0,02 & 0,26 & 1,75 \\
\hline 1922 & 0,97 & 0,50 & 0,03 & 1,02 & 3,07 \\
\hline 1923 & 0,91 & 0,61 & 0,01 & 1,43 & 3,88 \\
\hline 1924 & 1,38 & 1,99 & 0,05 & 4,27 & 6,88 \\
\hline 1925 & 1,10 & 2,48 & 0,02 & 2,48 & 5,93 \\
\hline
\end{tabular}

Sources : Commissariato generale dell'emigrazione (1926 : 1601-1604) ;

ACS, MI, DGSP (1882-1927) Giornali sanitari di bordo.

Alors que le flux vers les États-Unis était constitué de personnes jeunes, robustes et, en général, de chefs de famille qui voyageaient seuls et étaient soumis à des contrôles sévères aussi bien au départ qu'à l'arrivée, les courants migratoires en direction de l'Amérique du Sud comprenaient des proportions importantes de vastes secteurs d'émigrants "enrôlés" (Brunello, 1994; Franzina, 1979 et 1995). Les contrôles sanitaires à l'embarquement étaient pratiquement inexistants et il arrivait souvent que l'émigrant, auquel on ne donnait pas l'autorisation d'émigrer vers les États-Unis, décide de s'embarquer pour l'Amérique du Sud (Molinari, 2001).

Les publications médicales de l'époque regorgent de nombreux témoignages quant à la diffusion significative du trachome dans les colonies italiennes au Brésil. Emilio Padovani, I'un des médecins chargés par le Commissariat général de l'émigration de mener des recherches sur les conditions sanitaires des émigrants revenant des Amériques, dans sa tentative d'effectuer une première évaluation de l'étendue de la diffusion du trachome dans certaines zones du Brésil et de l'Argentine, considère la maladie comme une calamité et évalue les effets sur la santé des immigrés en termes de "carnage continuel» : 
"Alors qu'en Amérique du Nord les migrants atteints de trachome sont renvoyés suite aux contrôles médicaux, ceux débarquant en Amérique du Sud faute de dispositifs sanitaires équivalents échappent à toute surveillance épidémiologique. Ils sont internés dans

des fazendas où en l'absence d'hygiène et de traitements thérapeutiques adaptés, ils tombent facilement malades, constituant ainsi des foyers de trachome. Ces situations ont suscité l'indignation et la stupeur des visiteurs. " (Padovani, 1909 : 19)

Dans les journaux sanitaires de bord, la présence très fréquente de personnes atteintes de trachome hospitalisées dans les infirmeries et dans les locaux d'isolement parmi les rapatriés d'Amérique du Sud nous informe des niveaux de diffusion de la maladie et elle laisse supposer l'existence à bord de malades pour lesquels le trachome était arrivé à un stade maximum et irréversible. Sur le bateau Città di Torino, cette proportion de malades atteints de trachome parmi les rapatriés au cours d'un voyage de retour de Buenos Aires en 1906 est évaluée à $40 \%{ }^{9}$. Nous avons identifié des indications sporadiques sur la gravité de la situation sanitaire qui caractérise une grande partie de l'émigration italienne en Amérique du Sud dans les publications médicales de l'époque. Celles-ci, malgré la rareté des informations disponibles sur ce thème, soulignent la préoccupation que suscite l'étendue du trachome parmi les rapatriés et invitent à appliquer un contrôle sanitaire plus strict sur les migrants qui rentrent des pays de l'Amérique du Sud afin de limiter la contagion. Commentant les résultats des statistiques sanitaires de 1909, Teodorico Rosati, le médecin ayant dirigé les services de santé du Commissariat de l'émigration, met en garde contre la charge de morbidité de l'émigration de retour et note l'augmentation constante de malades du trachome en provenance du Brésil (Rosati, 1909).

L'état de désorganisation des services sanitaires concernant l'émigration aussi bien à terre qu'à bord, permet de dessiner grâce aux indicateurs généraux le tableau de l'étendue de l'enjeu sanitaire dans le cadre d'une migration de masse, mais ces données rendent difficilement compte des pathologies spécifiques. Les relevés statistiques font en effet référence aux maladies qui ont été diagnostiquées durant le voyage par le médecin du gouvernement ou par le commissaire de bord, ce qui exclut donc des comptages un certain nombre d'émigrants qui, pour de multiples raisons (la défiance envers le pouvoir médical, la crainte d'être refoulés en raison de leur état morbide dans le pays de destination ou hospitalisés une fois rapatriés), ne demandaient pas d'assistance sanitaire en dépit de leur situation. Une partie considérable du flux migratoire échappait aussi complètement à toute forme de contrôle sanitaire soit parce qu'ils embarquaient et débarquaient dans des ports à l'étranger, soit parce qu'ils voyageaient sur des bateaux dépourvus de service sanitaire, ou parce qu'ils embarquaient de manière semi-clandestine sous le regard complice des compagnies maritimes.

À partir de l'analyse des chiffres fournis par l'ensemble du corpus disponible entre 1903 à 1925 (archives et presse), nous observons la persistance de certaines maladies durant les allers, mais aussi les retours des Amériques. Bien qu'il soit impossible à partir de ces informations d'évaluer le rôle du flux transocéanique dans la diffusion en Italie de pathologies (pellagre, paludisme 
et tuberculose), soulignons que ces pathologies sont largement présentes dans les statistiques de morbidité. Le paludisme a les taux de prévalence les plus élevés, après la rougeole, durant les voyages vers I'Amérique du Nord et du Sud (voirTableau 4). L'importante diffusion des fièvres parmi les émigrants dans les voyages transocéaniques a été mise en relation avec l'intensification des flux migratoires en provenance de I'Italie méridionale par les responsables des statistiques sanitaires et par les médecins de bord (Corti, 1984).

Tableau 4 : Cas de paludisme déclarés durant les voyages transocéaniques en effectifs (1903-1925)

\begin{tabular}{|c|c|c|c|c|}
\hline & $\begin{array}{c}\text { Italie -Amérique } \\
\text { du Nord }\end{array}$ & $\begin{array}{l}\text { Amérique du } \\
\text { Nord - Italie }\end{array}$ & $\begin{array}{c}\text { Italie - Amérique } \\
\text { du Sud }\end{array}$ & $\begin{array}{l}\text { Amérique du } \\
\text { Sud - Italie }\end{array}$ \\
\hline 1903 & 86 & 28 & 34 & 29 \\
\hline 1904 & 61 & 49 & 64 & 11 \\
\hline 1905 & 194 & 24 & 110 & 17 \\
\hline 1906 & 197 & 48 & 98 & 21 \\
\hline 1907 & 160 & 58 & 114 & 50 \\
\hline 1908 & 32 & 48 & 104 & 20 \\
\hline 1909 & 70 & 20 & 60 & 33 \\
\hline 1910 & 102 & 55 & 158 & 55 \\
\hline 1911 & 58 & 57 & 19 & 23 \\
\hline 1912 & 78 & 40 & 30 & 28 \\
\hline 1913 & 133 & 43 & 70 & 16 \\
\hline 1914 & 35 & 37 & 8 & 30 \\
\hline 1915 & 2 & 15 & 1 & 16 \\
\hline 1916 & 17 & 6 & 1 & 6 \\
\hline 1917 & 2 & 1 & 0 & 0 \\
\hline 1918 & 0 & 0 & 0 & 5 \\
\hline 1919 & 7 & 4 & 52 & 2 \\
\hline 1920 & 23 & 0 & 44 & 6 \\
\hline 1921 & 58 & 7 & 51 & 5 \\
\hline 1922 & 12 & 7 & 62 & 6 \\
\hline 1923 & 15 & 4 & 93 & 26 \\
\hline 1924 & 13 & 4 & 102 & 18 \\
\hline 1925 & 7 & 2 & 69 & 33 \\
\hline
\end{tabular}

Sources : Commissariato generale dell'emigrazione (1926 : 1601-1604) ; ACS, MI, DGSP (1882-1927) Giornali sanitari di bordo.

Durant les voyages vers l'Amérique du Sud, nous remarquons un effectif important de malades atteints de trachome et de la gale, alors qu'au retour, les pathologies prévalentes sont le trachome et la tuberculose bien qu'avec des taux moins élevés, et I'ankylostomiase complètement absente des statistiques des voyages allers. Lors des rapatriements vers le Nord, les chiffres les plus élevés concernent la tuberculose pulmonaire, les aliénations mentales et le trachome. Cette dernière pathologie, bien que ne présentant pas de chiffres particulièrement élevés, est majoritairement plus répandue que lors des voyages allers. $\mathrm{Si}$, comme nous l'avons déjà remarqué, les contrôles sanitaires des émigrants au moment de l'embarquement en direction de l'Amérique du Sud étaient faits de manière peu consciencieuse, ils étaient complètement inexistants lors des voyages de retour. 
Dans une étude publiée en 1910, Ernesto Madia raconte ses expériences de voyage en tant que médecin et il se plaint du manque d'organisation au moment de l'embarquement à Rio de Janeiro et de l'état de confusion qui caractérise I'affluence des émigrants à bord des bateaux :

" II n'y a pas de discipline au moment de l'embarquement, les rapatriés montent dans le bateau à n'importe quelle heure et se dispersent dans le bâtiment sans que le médecin n'ait pu les ausculter auparavant. " (Madia, $1910: 453-474$ )

Les sources issues du service sanitaire de bord sont cependant les seules disponibles pour illustrer la gravité des conditions de santé des émigrants dans certaines zones du Brésil et de l'Argentine. Le médecin de bord du bateau Duca d'Aosta relate en septembre 1926 le voyage de retour de Buenos Aires à Gênes :

"Cinquante-deux passagers ont été contrôlés à l'embarquement pour des mutilations aux mains, des déformations physiques, des hernies inguinales, des problèmes de conjonctivites simples, trachomes ou cécité. $\aleph^{10}$

À partir des sources sanitaires à bord et des informations fournies par le journaliste médical, il s'avère que la mortalité infantile demeure constamment élevée durant les voyages transocéaniques. Un panorama statistique de la morbidité et de la mortalité infantile, effectué à partir de rapports sanitaires de voyages transocéaniques dont le nombre n'est pas précisé, est présenté dans un essai publié en 1900 dans la Rivista d'igiene e sanità pubblica :

"Les tableaux font référence au premier semestre de 1896 et indiquent des niveaux très élevés de morbidité et de mortalité parmi les enfants et les nourrissons : sur un total de 480 malades, 135 ont moins de cinq ans; sur un total de 137 décès enregistrés au cours des voyages transocéaniques examinés, quatre-vingt-dix sont des enfants "

(Druetti, $1890: 68$ )

Parmi les pathologies les plus répandues apparaissent la rougeole, la scarlatine et les maladies broncho-pulmonaires. Les mauvaises conditions hygiéniques du voyage par mer constituent le principal facteur explicatif de leur diffusion :

"Parmi les maladies de l'appareil respiratoire, les bronchites et les pneumonies sont les plus fréquentes. Elles se déclarent surtout pendant la saison froide et le plus souvent durant les voyages pour l'Amérique du Sud. La bronchite capillaire, bien que plus rare, contribue fortement aux taux de mortalité des petits enfants. ॥ (Druetti, 1890 : 68)

L'un des hygiénistes les plus importants de l'époque, Carlo Maria Belli, lors d'un bilan sur la situation sanitaire des émigrants pendant la période précédant I'approbation de la loi de 1901, avec peu d'éléments statistiques à sa disposition, calcule un taux de mortalité s'élevant à $40 \%$ parmi les émigrants transocéaniques, la mortalité infanto-juvénile est encore plus élevée:

"Le taux de mortalité infanto-juvénile le plus élevé (75\%) s'explique par les entérites, les bronchites, les pneumonies et les fièvres éruptives. " (Belli, 1905 : 287)

10 ACS, MI, DGSP (1910-1927) Giornali sanitari. Giornale sanitario del piroscafo Duca d'Aosta, b. 111. 
C'est l'organisation même des services sanitaires à bord qui semble expliquer l'augmentation du risque sanitaire existant, déjà très élevé, pour la santé des émigrants durant le voyage. L'habitude de faire sortir les émigrants des dortoirs aux premières heures de la matinée et de les laisser sur le pont à l'air libre durant toute la durée nécessaire au nettoyage et à la désinfection des dortoirs semblait accentuer le développement des maladies de l'appareil respiratoire. En outre, les substances utilisées pour accomplir la désinfection des locaux, le souffre et le chlorure de chaux, rendaient l'air des dortoirs irrespirable (Molinari, 1988).

Les caractéristiques des lieux pathogènes comme les dortoirs des émigrants se manifestaient de manière particulièrement évidente à l'occasion des maladies épidémiques. Ces espaces étaient sales, humides, malodorants, et infestés de punaises et de poux. Le médecin de bord du bateau Savoia écrit, en 1905, à propos d'une épidémie de rougeole qui s'était déclarée au cours d'un voyage de Gênes à Buenos Aires :

"Après les premiers cas, toutes les mesures hygiéniques possibles avaient été prises, mais évidemment l'infection s'était déjà déclarée compte tenu de la rapidité de propagation. Je ne pense pas me tromper en affirmant que c'est en raison de l'invasion des punaises dans les cales où dorment les émigrants. L'inconvénient de ces cales, malheureusement insurmontable, est qu'il est impossible d'ouvrir les hublots des cabines des ponts inférieurs, et qu'il est très compliqué de nettoyer en totalité les couchettes. " ${ }^{11}$

Les conditions des traversées entre le XIXe et le XXe siècle étaient telles que les navires prirent le caractère d'un lazaret. Un journaliste de l'époque a observé à propos de l'embarquement des migrants dans le port de Gênes :

"Le navire de Lazare est toujours là avec l'apparence d'un négrier et les yeux des malheureux qui attendent sont toujours effrayés, résignés à l'inconnu. » (Varaldo, $1916: 7$ )

\section{Migrants rapatriés et refoulés}

La raison la plus fréquente qui provoquait le "refoulement" vers le pays d'origine était la maladie. Sur ce thème, qui est peu connu de I'histoire des migrations historiques italiennes, nous avons quelques traces dans la documentation sanitaire de bord. Dans l'analyse des comptes rendus des commissaires et des journaux sanitaires de bord, nous pouvons constater que la tuberculose et la maladie mentale étaient les motifs principaux du rapatriement forcé ou du refoulement au moment du débarquement. En 1909, par exemple, sur un total de 700 migrants rapatriés malades des États-Unis, 400 étaient tuberculeux et 340 étaient "malades mentaux ${ }^{12}$. Plus de $70 \%$ de ces migrants avaient subi des sanctions de rapatriement forcé (Montano, 1910 : 11-30) (voir Tableau 5).

11 ACS, MI, DGSP (1882-1914) Giornali sanitari. Gionale sanitario piroscafo Savoia, b. 67.

12 ACS, MI, DGSP (1882-1936) Giornali sanitari di bordo, bb. 14-132. 
Tableau 5 : Italiens rapatriés car déclarés indigents par les autorités consulaires et par les organisations patronales en effectifs (1902-1925)

\begin{tabular}{|c|c|c|c|c|c|}
\hline & $\begin{array}{l}\text { États-Unis } \\
\text { d'Amérique }\end{array}$ & Brésil & Plata & Autres pays & Total \\
\hline 1902 & 393 & 1919 & 834 & 0 & 3146 \\
\hline 1903 & 627 & 1135 & 1223 & 0 & 2985 \\
\hline 1904 & 412 & 1770 & 1343 & 0 & 3525 \\
\hline 1905 & 534 & 2291 & 1708 & 0 & 4533 \\
\hline 1906 & 902 & 1789 & 1958 & 0 & 4739 \\
\hline 1907 & 2474 & 2074 & 1722 & 0 & 6270 \\
\hline 1908 & 3993 & 2201 & 1620 & 108 & 7922 \\
\hline 1909 & 3688 & 2594 & 2104 & 127 & 8513 \\
\hline 1910 & 4054 & 2377 & 1990 & 130 & 8551 \\
\hline 1911 & 4353 & 1700 & 3501 & 104 & 9748 \\
\hline 1912 & 4421 & 1505 & 3050 & 110 & 9086 \\
\hline 1913 & 3887 & 1680 & 3048 & 149 & 8764 \\
\hline 1914 & 5531 & 2202 & 3877 & 118 & 11528 \\
\hline 1915 & 4980 & 857 & 2032 & 130 & 7999 \\
\hline 1916 & 1883 & 331 & 1193 & 37 & 3444 \\
\hline 1917 & 669 & 78 & 405 & 7 & 1159 \\
\hline 1918 & 190 & 72 & 480 & 27 & 709 \\
\hline 1919 & 1120 & 216 & 494 & 56 & 1886 \\
\hline 1920 & 858 & 548 & 763 & 68 & 2237 \\
\hline 1921 & 3475 & 486 & 820 & 89 & 4870 \\
\hline 1922 & 2309 & 428 & 1057 & 43 & 3837 \\
\hline 1923 & 937 & 633 & 973 & 52 & 2595 \\
\hline 1924 & 922 & 920 & 1455 & 100 & 3397 \\
\hline 1925 & 664 & 872 & 1671 & 138 & 3345 \\
\hline
\end{tabular}

Source : Commissariato generale dell'emigrazione (1926: 1634).

Au début du XXe siècle, la tuberculose, surtout pulmonaire, devient très fréquente parmi les migrants italiens aux États-Unis (Molinari, 1988). En ce qui concerne la période 1903-1925, les statistiques sanitaires du Commissariat général de l'émigration - qui sous-estimaient pourtant le phénomène - signalent parmi ceux qui reviennent des États-Unis un nombre très important de tuberculeux et, dans une moindre mesure, de personnes présentant des troubles mentaux lors de leur retour en Italie. Parmi les rapatriés et les refoulés d'Amérique du Sud, nous trouvons également un nombre très important de personnes affectées par des trachomes, la tuberculose et des troubles mentaux (Molinari, 2014b).

Les comptes rendus des médecins de bord et les journaux sanitaires de bord confirment cette situation. Le médecin de La Gallia dans un compte-rendu sanitaire du 9 octobre 1902, après avoir exprimé de la bienveillance pour les bonnes conditions des passagers au cours du voyage de Gênes à New York, affirme : 
" Il n'en est pas de même pour le retour. Les navires sont fréquemment infectés en particulier par des tuberculeux et par d'autres personnes porteuses de diverses maladies chroniques, qui sont renvoyés vers leur patrie. $n^{13}$

Une situation semblable se vérifie la même année sur le paquebot Nord America :

"Au cours des voyages de retour, j'ai soigné de nombreux individus rapatriés en Italie parce qu'ils étaient affectés par des maladies chroniques et par des imperfections physiques donc inaptes au travail. Je les ai admis à l'embarquement au nom de la charité humaine, parce que la majeure partie d'entre eux avaient de la famille en Italie et n'avaient aucune ressource financière ni aucune relation ou connaissance en Amérique. $»^{14}$

Le médecin du Madonna dans son compte-rendu sur le voyage de retour de New York à Naples, en juillet 1905, observe :

"La tuberculose est la maladie la plus fréquente parmi les émigrants qui subissent des rapatriements des États-Unis, en particulier parmi ceux qui ont vécu à New York. 1 $^{15}$

Sur le bateau Hambourg, au cours du voyage de retour de NewYork à Gênes en février 1905, sur les sept migrants qui ont subi une sanction de rapatriement forcé, cinq sont touchés par une tuberculose pulmonaire; deux d'entre eux décèdent durant le voyage ${ }^{16}$. Parmi les migrants rapatriés des États-Unis ou refoulés au moment du débarquement en raison de maladie, beaucoup sont considérées comme "fous" (voir Tableaux 6 et 7).

De 1902 à 1920, les troubles mentaux arrivent en deuxième position juste après la tuberculose dans les statistiques sanitaires du Commissariat général de l'émigration. La majeure partie des migrants qui reviennent en Italie en tant qu' "aliénés mentaux" ont été refoulés lors de la visite médicale au moment du débarquement ou après avoir été internés dans des structures sanitaires ou d'assistance aux États-Unis.

Le nombre élevé des personnes rapatriés des États-Unis vers I'Italie pour maladies mentales s'explique par les conditions d'exploitation physique particulières, la ségrégation sociale des migrants italiens dans ce pays, et l'évolution de la psychiatrie. En effet, aux États-Unis, à partir de la première moitié du XIXe siècle, avec le développement du capitalisme, tous les mécanismes d'exploitation du marché international du travail se matérialisent d'une manière particulièrement efficace sur les migrants : exploitation intensive, discrimination institutionnalisée, exclusion de la participation politique et sociale (Frigessi Castelnuovo et Risso, 1982).

13 ACS, MI, DGSP (1882-1936) Giornali sanitari. Giornali sanitari del piroscafo Gallia, b. 132. 14 ACS, MI, DGSP (1900-1914) Giornali sanitari. Giornali del piroscafo Nord America, b. 98. 15 ACS, MI, DGSP (1882-1915) Giornali sanitari. Giornali sanitari piroscafo Madonna, b. 10. 16 ACS, MI, DGSP (1882-1915) Giornali sanitari. Giornali sanitari piroscafo Hambourg, b. 72. 
Tableau 6 : Incidence des maladies mentales lors des voyages retour transocéaniques (nouveaux cas pour mille voyageurs, 1903-1925)

\begin{tabular}{ccc}
\hline & $\begin{array}{c}\text { Amérique du } \\
\text { Nord }\end{array}$ & $\begin{array}{c}\text { Amérique du } \\
\text { Sud }\end{array}$ \\
\hline 1903 & 0 & 0 \\
1904 & 0,46 & 0,27 \\
1905 & 0,85 & 1,01 \\
1906 & 0,86 & 0,55 \\
1907 & 0,7 & 0,49 \\
1908 & 0,42 & 0,58 \\
1909 & 1,9 & 0,47 \\
1910 & 1,36 & 0,91 \\
1911 & 1,16 & 1,2 \\
1912 & 1,93 & 1,19 \\
1913 & 2,12 & 1,29 \\
1914 & 1,44 & 0,87 \\
1915 & 1,05 & 0,83 \\
1916 & 3,26 & 1,15 \\
1917 & 1,98 & 0,48 \\
1918 & 3,85 & 2,97 \\
1919 & 1,26 & 0,7 \\
1920 & 2,54 & 0,77 \\
1921 & 3,22 & 0 \\
1922 & 5,98 & 2,45 \\
1923 & 6,83 & 2,97 \\
1924 & 4,69 & 2,51 \\
1925 & 3,21 & 2,21 \\
\hline $59 r i a$ & & \\
\hline
\end{tabular}

Sources : Commissariato generale dell'emigrazione (1926 : 1607-1610) ; ACS, MI, DGSP (1900-1927) Giornali sanitari di bordo.

Tableau 7 : Rapatriés atteints de tuberculose, d'ankylostomasie et de trachome selon la région d'immigration en effectifs (1903-1909)

\begin{tabular}{|c|c|c|c|c|c|c|c|c|c|}
\hline & \multicolumn{3}{|c|}{ Paludisme } & \multicolumn{3}{|c|}{ Ankylostomasie } & \multicolumn{3}{|c|}{ Trachome } \\
\hline & $\begin{array}{c}\text { Amérique } \\
\text { du Sud }\end{array}$ & $\begin{array}{c}\text { Amérique } \\
\text { du Nord }\end{array}$ & Total & $\begin{array}{c}\text { Amérique } \\
\text { du Sud }\end{array}$ & $\begin{array}{c}\text { Amérique } \\
\text { du Nord }\end{array}$ & Total & $\begin{array}{c}\text { Amérique } \\
\text { du Sud }\end{array}$ & $\begin{array}{c}\text { Amérique } \\
\text { du Nord }\end{array}$ & Total \\
\hline 1903 & 48 & 179 & 227 & 7 & 0 & 7 & 0 & 12 & 12 \\
\hline 1904 & 77 & 278 & 355 & 10 & 2 & 12 & 15 & 10 & 25 \\
\hline 1905 & 77 & 369 & 446 & 18 & 1 & 19 & 4 & 22 & 26 \\
\hline 1906 & 81 & 457 & 538 & 16 & 1 & 17 & 87 & 52 & 139 \\
\hline 1907 & 110 & 518 & 628 & 15 & 15 & 30 & 29 & 9 & 38 \\
\hline 1908 & 139 & 495 & 634 & 25 & 25 & 50 & 238 & 0 & 238 \\
\hline 1909 & 135 & 505 & 640 & 8 & 8 & 16 & 332 & 72 & 404 \\
\hline
\end{tabular}

Sources : Rosati (1910 : 44) ; ACS, MI, DGSP (1900-1915), Giornali sanitari.

Au même moment où des flux migratoires de masse en provenance de l'Europe méridionale arrivent aux États-Unis, la psychiatrie s'affirme en tant que science. Son développement a supporté les théories de la non-assimilation d'une " new emigration " (Martellone, 1980 ; Luconi et Petrelli, 2008) qui par sa dimension et composition sociale apparaissait plus dangereuse que les courants 
migratoires du passé. Contrairement aux flux migratoires en provenance d'Europe du Nord, constitués de diverses classes sociales et qui s'étaient dilués sur une longue période dans la population américaine, les flux d'Italiens qui arrivent en masse aux États-Unis entre la fin du XIXe et le début du XXe siècle étaient composés principalement de paysans pauvres (Corti, 2007 et 2013). De grands noms de la médecine et de la psychiatrie légitiment d'un point de vue scientifique les préjudices à l'encontre les nouveaux émigrants causés par la société américaine. Par exemple, le psychologue Robert M. Yerks de I'université de Harvard, commente le rapport entre comportements dit "anormaux" et dégénération de la race :

"Comment peut-il y avoir une égalité sociale quand il y a différents niveaux d'intelligence entre les personnes? Les hommes à la peau plus foncée de l'Europe méridionale et les Slaves de l'Europe orientale sont moins intelligents que les hommes à la peau claire d'Europe du Nord et occidentale; dans le classement de l'intelligence, le nègre est à la dernière place. ${ }^{17}$ (Moriani, 1999 : 56)

En 1907, parmi les collaborateurs de la Commission Dillingham instituée par le gouvernement des États-Unis pour préparer les mesures qui limiteront les flux migratoires d'Europe du Sud, un rôle important a été joué par Harry Hamilton Laughlin, I'un des plus grands partisans des théories eugéniques. Dans le rapport présenté à la Commission en 1922, Analysis of American's modern melting pot, Laughlin affirme que c'est un "suicide racial» pour la civilisation américaine de laisser entrer aux États-Unis des individus qui proviennent de groupes génétiquement "inférieurs" comme les Italiens, les Juifs et les Espagnols (Laughlin, 1923).

Aux États-Unis, les migrants qui présentaient des troubles mentaux et s'adressaient aux établissements de santé et de soins couraient le risque d'être rapatriés. La législation sur l'immigration contenait des règles qui interdisaient l'entrée aux personnes "mentalement perturbées". En 1882, le premier décret fédéral interdisant le débarquement des aliénés et des handicapés mentaux et le rapatriement forcé pour les délinquants fut adopté. Toujours selon cette loi, le migrant qui, un an après son arrivée aux États-Unis, avait eu recours à des établissements de soins psychiatriques était "refoulé " dans son pays d'origine. Un décret de 1903 sur l'immigration, interdisait le débarquement des épileptiques et de ceux qui avaient été hospitalisés pour maladies mentales au cours des cinq années précédentes. En 1907, ce décret a été intégré à un ensemble de lois restrictives qui établissaient le refoulement de ceux qui semblaient inaptes au travail au moment même du débarquement. Un décret de 1917 précise les maladies mentales interdisant l'entrée sur le territoire : peuvent être refoulés les psychopathes, les alcooliques, les frénétiques et les "agités" (Neuman, 1996).

Parmi les migrants refoulés ou rapatriés pour maladie mentale, seule une petite partie était internée dans des hôpitaux psychiatriques lors de leur retour en Italie. À partir de 1904, la première loi organique sur le fonctionnement des hôpitaux psychiatriques italiens prévoit des procédures d'assistance et de

17 Robert Means Yerkes fut, avec Carl Brigham, parmi les fondateurs de la psychométrie et parmi les plus grands partisans aux Etats-Unis de la médecine eugénique. 
contrôle concernant les migrants rapatriés pour maladie mentale ${ }^{18}$. II s'agissait, toutefois, de lois difficiles à appliquer en raison du manque d'établissements de soins aux migrants dans les ports (Molinari, 2002). À partir des histoires des patients internés dans des cliniques psychiatriques publiées dans certaines revues psychiatriques, nous constatons que l'internement dans les hôpitaux psychiatriques s'effectuait, généralement, après des périodes plus ou moins longues après le retour du migrant dans son pays d'origine. L'étude des principales revues italiennes de psychiatrie et de neuropsychiatrie entre le XIXe et $X X e$ siècle met en évidence le peu d'attention des milieux médico-psychiatriques italiens aux pathologies mentales des migrants rapatriés. Le rapport entre migrations et maladie mentale était alors peu étudié. Nous avons trouvé un article publié en 1889 dans la revue Archives italiennes des maladies nerveuses et plus particulièrement des troubles mentaux, et un autre datant de 1909, publié dans Notes et revues de psychiatrie. II s'agit de brefs comptes rendus qui présentent des statistiques relatives à l'internement des migrants dans les hôpitaux psychiatriques de Côme, Pesaro, Ferrare (Molinari, 2010 : 59).

Le rapatriement des migrants "fous" suscite, surtout dans les milieux psychiatriques de l'époque, une inquiétude concernant la difficulté d'exercer des formes de contrôle sur des sujets qui, en tant que "déviants", sont considérés comme socialement dangereux. Certains articles publiés dans la Rivista di emigrazione sont, à ce sujet, très significatifs ${ }^{19}$. Dans l'un d'entre eux, Antonio D'Ormea, directeur de I'hôpital psychiatrique de Pesaro, constatant qu'après leur débarquement les migrants atteints de maladies mentales échappaient à toute forme de contrôle, insiste sur la nécessité de mettre en œuvre des formes de prévention sociale pour séparer et isoler des individus qui pourraient contaminer le "corps sain" du pays :

"Un grand nombre d'entre eux apparaissent, déjà quand ils partent, comme des dégénérés qui dans la recherche fébrile et souvent irréfléchie de l'inconnu, font preuve d'un certain déséquilibre ou d'une certaine carence d'esprit critique. Tous ces gens-là devraient être éloignés des aléas d'une vie qui ne peut qu'augmenter cette insuffisance du système nerveux et les fera succomber avant d'arriver à leur destination ou bien après une brève lutte qui les ramènera à leur patrie, vaincus et inutiles à eux-mêmes et aux autres. " (D'Ormea, $1902: 1-7)$

Déjà en 1891, un autre psychiatre, Rodrigo Fronda, s'interrogeait à propos de la diffusion de la maladie mentale parmi les migrants rapatriés des États-Unis :

\footnotetext{
18 Le règlement exécutif de la loi du 14 février 1904 sur les hôpitaux psychiatriques et sur les aliénés prévoit que le migrant rapatrié pour trouble mental est confié à l'autorité de sécurité publique qui avait la charge de trouver un internement provisoire. Dans les trois jours suivant le débarquement, les autorités de sécurité publique devaient en référer aux autorités judiciaires, lesquelles devaient établir à quelle structure psychiatrique était destiné le migrant. C'était le médecin de bord qui devait s'occuper de ces dossiers; il devait se charger de contacter les médecins des ports de débarquement qui, à leur tour, devaient prévenir les autorités de sécurité publique.

19 La Rivista di emigrazione naît en 1908 et est soutenue, même financièrement, par les milieux économiques les plus philo-immigrationnistes; elle est imprimée à Pesaro. Parmi les collaborateurs : P. Ascoli, directeur de l'École supérieure de commerce, A. Alberti, directeur de I'hôpital psychiatrique de Pesaro, A. Cabrini, responsable du bureau d'émigration de la Société humanitaire, A. Mosso et P. Cogliolo et N. Colajanni, médecins et académiciens, U. Conti, responsable de I'Institut des études coloniales et G. Mortara, économiste.
} 
"L'émigration serait-elle la cause première du mal?" (Fronda, $1891: 10-14)$. Pour trouver une réponse, il effectue une enquête sur les migrants internés au cours des trois dernières années (1888-1890) dans I'hôpital psychiatrique de Nocera Inferiore (Naples) : il s'agit de cinquante-quatre hommes et six femmes en provenance des États-Unis. D'après les informations qu'il réussit à obtenir à partir des dossiers médicaux, Fronda acquiert la conviction que les migrants qui "perdent la raison" sont des sujets prédisposés à la folie en raison de "leur propre constitution ". Leur penchant à la mobilité apparaît déjà à Fronda comme un symptôme de perversité mentale :

"Celui qui pense à émigrer se trouve déjà mal à l'aise dans l'environnement où il vit, parce que son insuffisance qui s'exprime par la misanthropie, l'irritabilité, la susceptibilité et la méfiance, lui enlève les joies que vivent les êtres moins imparfaits. Par conséquent,

cet individu attribue tout le mal qu'il éprouve à l'environnement qui l'entoure, ainsi il

développe en lui les racines d'un délire de persécution. II s'éloigne donc de son envi-

ronnement pour chercher des milieux plus favorables et, quand il arrive dans le pays étranger, souvent il vit les plus grandes privations pour accumuler de l'argent et dans des conditions climatiques ou, même, les vices auxquels il s'abandonne font en sorte que les conditions de son pauvre cerveau malade s'aggravent. Le délire qui auparavant se manifestait de temps en temps, arrive alors à sa plénitude et, peu à peu, se structurant va jusqu'à s'exprimer avec puissance. " (Fronda, 1891 : 6)

Nous trouvons une confirmation du rôle du psychiatre dans la validation du stéréotype du migrant comme sujet "déviant", dans les articles sur les maladies des migrants publiés dans la Rivista di emigrazione. Le psychiatre Padovani, directeur de l'hôpital psychiatrique de Ferrara, prend en charge cette rubrique et ce n'est pas un hasard si la revue donne plus d'espace à la question des pathologies mentales par rapport à d'autres maladies bien plus répandues (tuberculose, trachome). Dans un article de 1911, après avoir mis en relief le fait qu'entre 1906 et 1909, 639 émigrants aliénés étaient rentrés des États-Unis, Padovani se demande :

" Où sont passés ces 639 aliénés, qui ne représentent pas toutefois notre douloureuse masse de malades, car nous n'avons pas les chiffres réels des émigrants rapatriés sur les paquebots, où le commissaire gouvernemental était absent, et il nous manque le nombre d'émigrants débarqués dans les ports à l'étranger? Nous sommes dans l'ignorance la plus complète concernant le nombre de ces malades, nous ne savons pas si et dans quels hôpitaux psychiatriques ils ont été internés. " (Padovani, 1911 : 7-19)

Dans d'autres articles, toujours dans la Revue du Commissariat, Padovani analyse les maladies mentales les plus répandues enregistrées dans les statistiques sanitaires de bord. II écrit à propos de la diffusion de la frénésie alcoolique:

" Nous constatons qu'au cours des deux années 1904-1906, le nombre de personnes alcooliques de retour des Amériques a triplé. Nous devons penser au danger que cette masse de rapatriés représente à la fois pour eux-mêmes [...], mais aussi pour les tendances vicieuses qu'elle peut provoquer chez nos paysans à travers le mauvais exemple." (Padovani, 1912: 321-329)

En ce qui concerne les migrants refoulés des États-Unis à cause d'une maladie mentale, les médecins de bord se limitaient à confirmer le diagnostic 
qui apparaissait dans les dispositions d'expulsion. Le fait qu'un migrant puisse être refoulé au moment du débarquement et rapatrié comme aliéné mental était inclus dans l'exercice du pouvoir discrétionnaire des autorités américaines. II suffisait d'un malaise dû à la fatigue du voyage ou d'un état de confusion provoqué par les procédures bureaucratiques de contrôle dans les ports de débarquement, pour que la procédure de "refoulement" soit appliquée. Les comptes rendus des médecins de bord confirment cette pratique de routine des voyages d'émigration. Nous pouvons lire par exemple dans une expertise sanitaire de 1908 :

"Parmi les 100 Italiens refoulés depuis le port de New York en tant qu'aliénés, dix étaient déjà aliénés dans leur patrie, quarante-cinq avaient donné des signes d'irritation pendant le voyage, quatre étaient ivres, trente paraissaient bizarres aux médecins parce qu'ils gesticulaient, cinq avaient déclaré avoir des parents lointains internés dans des hôpitaux psychiatriques. $\Perp^{20}$

Nous ne disposons pas d'informations fiables pour étudier ce qui arrivait aux migrants qui revenaient en Italie avec des problèmes de santé mentale. II est probable que ceux qui réussissaient à retrouver leur propre famille échappaient, au moins temporairement, à l'internement psychiatrique. La situation était bien différente pour ceux qui ne pouvaient pas compter sur un réseau familial. Si tel était le cas, les médecins des ports s'adressaient aux autorités de Sécurité publique qui se chargeaient d'interner les migrants dans des hôpitaux psychiatriques.

Cependant, une toute autre approche que celle qui dominait le milieu psychiatrique de l'époque apparaît dans deux enquêtes sur le rapport entre migration et maladie mentale publiées dans la revue de l'hôpital psychiatrique de Nocera Inferiore, II Manicomio Moderno. Francesco Lener est l'auteur de la première, Le malattie mentali e le correnti migratorie nell'Italia meridionale (1908 et 1909) et Giuseppe Tolone, médecin de I'hôpital psychiatrique de Girifalco (Cosenza), de la seconde, Emigrazione e follia (1909). Les auteurs de deux études, notamment Lener, considèrent la maladie mentale comme un effet de l'exploitation économique et de l'isolement social des migrants chez eux comme à l'étranger. Lener examine les quatre-vingt-sept migrants internés à Nocera Inferiore entre 1905 et 1907 et publie les rapports cliniques. Les internés examinés représentent $53 \%$ des admis à l'hôpital psychiatrique au cours de ces trois années et les diagnostics les plus fréquents sont ceux de frénésie alcoolique et de démence précoce. Quatre-vingt-cinq de ces migrants ont été rapatriés ou refoulés après leur débarquement aux États-Unis : il s'agissait d'hommes, en grande partie des paysans, d'un âge compris entre dix-neuf et cinquante-cinq ans; aux États-Unis, la plupart d'entre eux avaient effectué des travaux pénibles et sans qualification : maçons, mineurs, terrassiers, porteurs. Comme le note Lener, l'expérience migratoire a joué un rôle décisif dans la manifestation de la maladie mentale :

" ll y a trois facteurs qui contribuent de manière importante au développement de problèmes mentaux parmi les migrants : I'alcoolisme, la syphilis et le travail. "

(Lener, $1908: 210$ )

20 ACS, MI, DGSP (1901-1912) Relazione sanitaria del piroscafo "Città di Torino", b. 52. 
À partir des résumés des rapports cliniques présentés par Lener émerge une uniformité d'expériences et de "destins" migratoires : travaux pénibles, isolement social et solitude allégés par la consommation d'alcool. Les internés sont des hommes qui ont dû affronter seuls l'expérience migratoire et beaucoup d'entre eux ont effectué des travaux manuels dans des régions du nord-est des États-Unis, comme le New Jersey et la Pennsylvanie. Sur quatre-vingt-sept migrants internés, trois seulement semblent avoir trouvé des opportunités de travail qui leur ont permis d'accumuler un petit capital. Dans presque tous les cas, on signale l'abus d'alcool, qui pour un nombre significatif (vingt) s'accompagne de la contagion par la syphilis. L'histoire de I. D. illustre parfaitement cette situation. I. D. est un paysan qui émigre à l'âge de dix-sept ans :

" II est resté en Amérique du Nord pendant cinq ans. II a consommé de l'alcool et a contracté la syphilis. II a travaillé dans des mines. Il a commencé à donner des signes de déséquilibre et a été renvoyé en Italie. II a été interné dans un hôpital psychiatrique cinq jours après son rapatriement. Hérédité négative. " (Lener, $1908: 210)$

Même si elles sont très synthétiques, ces histoires laissent entrevoir des vies très difficiles. Ces migrants ne semblent pas avoir tissé dans le pays de destination des liens avec des parents ou des compatriotes. Et, peut-être qu'en raison des difficultés engendrées par la réalisation de leur projet migratoire, ils ont maintenu de faibles rapports avec les familles et les parents qu'ils ont laissés dans leur patrie. Selon les rapports, seuls deux d'entre eux ont été rapatriés à la demande de parents. Dans la plus grande partie des cas, l'internement dans les hôpitaux psychiatriques est survenu peu de temps après le rapatriement. Ceux qui revenaient chez eux sans ressource économique et avec des problèmes mentaux devenaient un "poids" pour la famille. L'expérience migratoire marque une rupture des liens et des réseaux sociaux que seul le succès d'un projet migratoire aurait pu, en partie, atténuer.

II n'était pas toujours facile de différencier les migrants des autres internés dans un hôpital psychiatrique ce qui ne permet pas d'identifier le rapport entre développement de la maladie et expérience migratoire. Ce problème est mis en exergue par Giuseppe Tolone dans une étude sur les migrants internés dans I'hôpital psychiatrique de Girifalco (Cosenza) (Greco, 2018) au cours des années 1903-1906 :

"Je suppose de manière sensée que le nombre de migrants internés est supérieur à celui que j'ai relevé. Mais souvent, malheureusement, on ne sait que peu ou rien sur les internés. Beaucoup d'entre eux sont conduits à l'hôpital psychiatrique par des personnes qui les connaissent peu ou pas du tout et nous n'obtenons que très peu d'informations à travers les certificats incomplets et de mauvaise qualité qui les accompagnent. "

(Tolone, $1908: 34$ )

Tolone fournit quelques informations sur les évènements migratoires des internés :

"Les cinquante-six cas sont tous de retour de l'Amérique du Nord, comme le montre l'anamnèse de chaque tableau nosologique. Certains devinrent fous pendant le voyage d'aller et furent donc renvoyés dans leur pays; d'autres, dès leur arrivée, furent internés dans des hôpitaux psychiatriques étrangers puis ensuite rapatriés. " (Tolone, 1908 : 45) 
Comme Lener l'avait déjà constaté pour l'hôpital psychiatrique de Nocera Inferiore, dans celui de Girifalco les migrants représentent également une partie importante des internés dans les premières années du XXe siècle : $24 \%$ dans la période de 1903 à 1906. Les internés sont tous des migrants de sexe masculin d'un âge compris entre dix-neuf et quarante-cinq ans. Contrairement aux migrants internés à Nocera Inferiore, qui sont presque tous paysans, ceux de Girificalco sont surtout des artisans (cordonniers, menuisiers, couturiers). Le nombre de migrants refoulés au moment du débarquement ou ayant subi des formes de rapatriement forcé est très élevé.

Les rapports cliniques recueillis par Tolone restituent des fragments d'une humanité particulièrement pauvre et souffrante. Le traumatisme intrinsèque de l'expérience migratoire, à la fois comme rupture des liens affectifs et sociaux que comme incertitude concernant leur destin, apparaît de manière très nette dans certaines histoires cliniques. Parmi celles-ci, retenons celle d'un jeune qui, en 1903, part pour les États-Unis :

"Venanzio C., dix-neuf ans, célibataire, cordonnier. II se rend à Baltimore pour trouver un emploi, mais pendant le voyage, craignant d'être refoulé par les autorités parce que personne ne l'accompagnait, il commence à donner des signes d'aliénation mentale si bien qu'on l'empêche de débarquer et qu'il fut rapatrié. La famille, qui s'était rendue à Naples pour le rencontrer, le conduisit immédiatement ici. Toujours triste et solitaire. Diagnostic : frénésie dépressive. Hérédité négative. » (Tolone, 1908 : 53)

Dans beaucoup de cas, l'échec du projet migratoire au moment du débarquement provoque une réaction mentale qui évolue de manière pathologique. Sur les cinquante-trois émigrants interrogés par Tolone, une grande partie d'entre eux a été refoulée au débarquement. Certaines histoires cliniques illustrent le traumatisme de ceux qui voyaient échouer leur projet migratoire dès leur arrivée. C'est le cas d'un couturier, marié et père de trois enfants qui émigre, en 1905, aux États-Unis :

"En novembre dernier, Domenico G. se rendit à New York, mais quand il arriva, on ne I'autorisa pas à débarquer, on ne sait pas pour quelle raison. De retour dans son pays, il commença à ne plus avoir envie de travailler, ensuite il ne voulut plus sortir de chez lui,

il mangeait peu. Il fut donc interné dans un hôpital psychiatrique. Le malade est accueilli dans un état de dépression extrême. II est aboulique, inactif, il déchire souvent ses vêtements. Diagnostic : état mélancolique. Hérédité négative " (Tolone, 1908 : 41).

GiuseppeTolone, tout comme Francesco Lener, considère la maladie mentale des migrants comme une conséquence des traumatismes économiques et sociaux subis tant dans les lieux de départ que ceux d'arrivée. Seul le migrant qui trouve des "réseaux" de soutien dans les pays de destination peut survivre sans tomber dans la misère et la folie :

"Les plus forts sont ceux qui rencontrent des situations qui les rendent forts. Ce sont les plus chanceux. " (Tolone, 1908 : 37)

Nous avons peu de documents qui illustrent les traumatismes physiques et psychiques vécus par les masses de migrants dans les années où la migration transocéanique s'amplifie. Mis à part quelques hâtives et synthétiques certifications des maladies reportées sur les journaux de bord sanitaires et sur les 
revues médicales du début du XXe siècle. À l'époque comme aujourd'hui, le migrant est surtout un "corps au travail" qui, quand il tombe malade, devient un "corps inutile", à la fois pour la société de destination et pour celle d'origine. L'hôpital psychiatrique a joué, d'un point de vue historique, le rôle de "dépôt" de personnes considérées comme des corps socialement inutiles. Dans le cas des migrants, comme l'indiquent les articles publiés dans la revue II Manicomio Moderno, la fonction de ségrégation et d'aliénation de l'individu à travers l'internement assume un caractère particulièrement punitif. Quand le migrant, contrairement à d'autres internés, rentre à l'hôpital psychiatrique, il laisse derrière lui deux vies : celle qu'il a trouvée et celle, qu'en vain, il a cherchée ailleurs.

\section{Références bibliographiques}

Arru Angelina e Ramella Franco (Eds.) (2003) L'Italia delle migrazioni internazionali. Donne, uomini, mobilità in età moderna e contemporanea, Roma, Donzelli, $390 \mathrm{p}$.

Belli Carlo Maria (1905) Igiene navale. Manuale per i medici di bordo, ufficiali, naviganti e costruttori navali, Milano, Treves, 23 p.

Brunello Piero (1994) Pionieri: gli italiani in Brasile e il mito della frontiera, Roma, Donzellli, 123 p.

Campesi Giuseppe (2013) La detenzione amministrativa degli stranieri. Storia, diritto, politica, Roma, Carocci, 247 p.

Commissariato generale dell'emigrazione (1926) Annuario statistico dell'emigrazione italiana dal 1876 al 1923, Roma, Ed Consolato Generale dell'emigrazione, $1720 \mathrm{p}$.

Corti Paola (2013) Temi e problemi di storia delle migrazioni internazionali, Viterbo, Sette Città, $131 \mathrm{p}$.

Corti Paola (2003) Storia delle migrazioni internazionali, Roma-Bari, Laterza, $146 \mathrm{p}$.

Corti Paola (1984) Malaria e povertà contadina nel Mezzogiorno, in Franco Della Peruta Ed., Medina e malattia. Storia d'Italia. Annali 7, Torino, Einuadi, pp. 663-678.

Cuttitta Paolo (2012) Lo spettacolo del confine. Lampedusa tra produzione e messa in scena della frontiera, Milano, Mimesis, $127 \mathrm{p}$.

Dal Lago Alessandro (2003) Polizia globale. Guerre e conflitti dopo I'11 settembre, Verona, Ombre corte, $134 \mathrm{p}$.

Druetti Giuseppe (1890) Sullo stato sanitario degli emigranti nelle traversate transoceaniche, Rivista di igiene e di sanità pubblica, 3 (199), pp.59-70.

Franzina Emilio (1995) Gli italiani al Nuovo Mondo: l'emigrazione italiana in America 1492-1942, Milano, Mondadori, 644 p.

Franzina Emilio (1979) Merica! Merica!: emigrazione e colonizzazione nelle lettere dei contadini veneti in America Latina 1876-1902, Milano, Feltrinelli, 229 p.

Frigessi Castelnuovo Delia e Risso Michele (1982) A mezza parete. Emigrazione, nostalgia, malattia mentale, Torino, Einaudi, 212 p. 
Fronda Roderigo (1891) La pazzia negli emigranti. Nota preventiva, I/ Manicomio Moderno, a. V, 2, pp. 2-17.

Greco Oscar (2018) I demoni del mezzogiorno. Follia, pregiudizio e marginalità nel manicomio di Girifalco (1881-1921), Soveria Manneli, Rubettino, 256 p.

Kanstroom Daniel (2007) Deportation Nation: Outsider in American History, Cambridge, MA, Harvard University Press, 340 p.

Laughlin Harry H. (1923) Analysis of American's modern melting pot. Testimony before the House Committee on Immigration and Naturalisation. House of Representatives, 21 November 1922, Washington DC, US Government Printing Office, $459 \mathrm{p}$.

Lener Francesco (1909) Les maladies mentales et les courants migratoires dans I'Italie méridionale, II Manicomio Moderno, 21 (21), pp. 177-186.

Lener Francesco (1908) Le malattie mentali e le correnti migratorie nell'Italia meridionale, II Manicomio Moderno, 20 (18), pp. 183-218.

Leogrande Alessandro (2015) La frontiera, Milano, Feltrinelli, 316 p.

Luconi Stefano e Petrelli Matteo (2008) L'immigrazione italiana negli Stati Uniti, Bologna, II Mulino, $214 \mathrm{p}$.

Madia Ernesto (1910) Studio di indole igienica: trentotto viaggi in servizio di emigrazione, Annali di medicina navale e coloniale, 6 (1), pp. 453-474.

Martellone Anna Maria (Ed.) (1980) La "questione" dell'immigrazione negli Stati Uniti, Bologna, II Mulino, 334 p.

Molinari Augusta (2014a) Les migrations italiennes au début du XXe siècle. Le voyage transocéanique entre évènement et récit, Torino/Paris, L'Harmattan, $218 \mathrm{p}$.

Molinari Augusta (2014b) De emigrantes a enfermos mentales. Historias de la emigración transoceánica italiana, Estudios migratorios latinoamericanos, 27/28 (75-76), pp. 3-21.

Molinari Augusta (2010) Emigrazione e follia nel primo Novecento, Rivista sperimentale di freniatria, 134 (3), pp. 47-65.

Molinari Augusta (2005) Traversate. Vite e viaggi dell'emigrazione transoceanica italiana, Milano, Selene, 140 p.

Molinari Augusta (2004) Aspetti sanitari dell'emigrazione transoceanica italiana, História. Debates e tendências, 1, pp. 205-224.

Molinari Augusta (2001) Porti, trasporti e compagnie, in Piero Bevilacqua, Andreina De Clementi e Emilio Franzina Eds., Storia dell'emigrazione italiane, Vol. 1 Partenze, Roma, Donzelli, pp. 237-255.

Molinari Augusta (1993) Emigration traffic in the port of Genoa between the Nineteenth and Twentieth Centuries: Shipping and Problems of Social Hygiene, Journal of American Ethnic History, 1, pp. 100-118.

Molinari Augusta (1990) Fuentes para la Historia de la Emigración Transoceanica italiana: la documentación sanitaria de a bordo, Estudios migratorios latinoamericanos, 20 (15-16), pp. 533-545. 
Molinari Augusta (1988) Le navi di Lazzaro. Aspetti sanitari dell'emigrazione transoceanica itali : il viaggio per mare, Milano, Franco Angeli, 274 p.

Montano Antonio (1910) Statistica degli emigranti ricoverati nelle infermerie di bordo nell'anno 1909, Bollettino dell'emigrazione, 26.

Moriani Gianni (1999) II secolo dell'odio. Conflitti razziali e di classe nel Novecento, Venezia, Marsilio, 262 p.

Neuman Gerald L. (1996) Strangers to the Consitution: Immigrants, Borders and Fundamental Law, Princeton, Princeton University Press, 283 p.

Orazi Stefano (2015) "I am hungry, I am Italian". Il problema del lungo esodo nella "Rivista di emigrazione", Roma, Istituto per la storia del Risorgimento italiano, $469 \mathrm{p}$.

Ormea Antonio d' (1902) Per la profilassi psichica dei nostri emigranti, Rivista di emigrazione, a. II, 2, pp. 1-7.

Ostuni Maria Rosaria (2001) Leggi e politiche di governo, in Piero Bevilacqua, Andreina De Clementi e Emilio Franzina Éds., Storia dell'emigrazione italiana, Vol. 1 Partenze, Roma, Donzelli, pp. 309-319.

Padovani Emilio (1912) Epilessia e alcolismo nell'emigrazione transoceanica italiana, Rivista di emigrazione, a. V, 1, pp. 321-329.

Padovani Emilio (1911) Sulla necessità di una statistica degli emigranti rimpatriati e respinti dall'America, Rivista di emigrazione, a. IV, 4, pp. 7-19.

Padovani Emilio (1909) Le condizioni igienico-sanitarie dell'emigrazione transoceanica italiana: II tracoma, Rivista di emigrazione, 2 (1), pp. 5-26.

Rosati Teodorico (1910) II servizio igienico sanitario nell'emigrazione transoceanica per l'anno 1909, Roma, Ed. Commissariato generale dell'emigrazione, 130 p.

Rosati Teodorico (1909) Assistenza sanitaria degli emigranti e dei marinai, Milano, Vallardi, $311 \mathrm{p}$.

Schmoll Camille, Thiollet Hélène et Wihtol de Wenden Catherine (Éds.) (2015) Migrations en Méditerranée. Permanences et mutations à l'heure des révolutions et des crises, Paris, CNRS Éditions, 362 p.

Sori Ercole (1979) L'emigrazione italiana dall'Unità alla prima guerra mondiale, Bologna, II Mulino, $512 \mathrm{p}$.

Sossi Federica (2005) Storie migranti. Viaggi tra i nuovi confini, Roma, Derive Approdi, $163 \mathrm{p}$.

Tichenor Daniel J. (2002) Dividing Lines: The Politics of Immigration Control in America, Princeton, Princeton University Press, $378 \mathrm{p}$.

Tolone Giuseppe (1908) Emigrazione e follia, II Manicomio Moderno, 20 (19), pp. 29-66.

Varaldo Alessandro (1916) I/ porto di Genova, Milano, Treves, 24 p. 


\section{Augusta Molinari}

\section{Les migrations transocéaniques italiennes au début du $X X e$ siècle : un autre regard}

Les voyages dramatiques actuels des migrants pour traverser les frontières par la mer nous imposent un nouveau regard sur l'historiographie des migrations transocéaniques italiennes du XXe siècle. À cette époque les traversées étaient moins dangereuses, mais elles n'étaient pas pour autant sans risques. En raison du manque d'assistance médicale et des mauvaises conditions d'hygiène, les migrants tombaient souvent malades ou pouvaient mourir pendant le voyage. Par ailleurs, la maladie était la principale cause de refoulements dans les ports d'arrivée et de rapatriement forcé, notamment aux États-Unis. L'analyse des différentes sources (documents sanitaires rédigés à bord des bateaux, revues médicales, rapports de commissaires) permet de saisir la complexité de ce phénomène et de comprendre comment déjà à cette époque, les projets des migrants pouvaient échouer avant même qu'ils n'atteignent leur destination.

\section{Italian Transoceanic Migration at the Beginning of the 20th Century: A Different Look}

The current dramatic journeys of migrants to cross borders by sea require us to take a new look at the historiography of Italian transoceanic migration in the 20th century. At that time, the crossings were less dangerous, but they were not without risks. Due to lack of medical assistance and poor hygiene conditions, migrants often became ill or could die during the trip. In addition, the disease was the main cause of expelling in ports of arrival and return migration, particularly in the United States. The analysis of the various sources (health documents written on board ships, medical journals, commissioners' reports) makes it possible to understand the complexity of this phenomenon and to understand how, even at that time, migrants' projects could fail even before they reached their destination.

\section{La migración transoceánica italiana a principios del siglo $X X$ : una otra mirada}

Los dramáticos viajes actuales de los migrantes para cruzar las fronteras por mar nos obligan a revisar la historiografía de la migración transoceánica italiana en el siglo XX. En ese momento los cruces eran menos peligrosos, pero no estaban exentos de riesgos. Debido a la falta de asistencia médica y a las malas condiciones de higiene, los migrantes a menudo se enfermaban o podían morir durante el viaje. Además, la enfermedad es la principal causa de devolución en los puertos de llegada y de repatriación forzosa, en particular en los Estados Unidos. El análisis de las distintas fuentes (documentos de salud escritos a bordo de los buques, revistas médicas, informes de los comisarios) permite comprender la complejidad de este fenómeno y cómo, incluso en ese momento, los proyectos de los migrantes podían fracasar incluso antes de llegar a su destino. 